\title{
Effects of Magnet Errors in the ILC 14 mrad Extraction Line*
}

\author{
Dragan Toprek \\ VINCA Institute of Nuclear Sciences, 11001 Belgrade, Serbia ${ }^{\dagger+}$ \\ The Cockcroft Institute, University of Manchester, UK ${ }^{\ddagger \ddagger}$ \\ Yuri Nosochkov \\ SLAC National Accelerator Laboratory, 2575 Sand Hill Road, Menlo Park, CA 94025, USA
}

\begin{abstract}
The ILC baseline extraction line is designed for 14 mrad horizontal crossing angle between $\mathrm{e}^{+}$and e- colliding beams at Interaction Point (IP). The extraction optics in the Interaction Region (IR) includes a detector integrated dipole field (anti-DID) to reduce orbit perturbation caused by the detector solenoid and minimize detector background. This paper presents a study of random field and alignment errors in the extraction magnets, compensation of the induced orbit perturbation, and effects of errors on extraction beam power loss. The results are obtained for the baseline ILC energy of $500 \mathrm{GeV}$ center-of-mass and three options of beam parameters.
\end{abstract}

* Work supported by the U.S. Department of Energy contract DE-AC02-76SF00515 and by the Cockcroft Institute, University of Manchester, UK.

†† Author's present address.

\# Author's affiliation when this study began. 


\title{
Effects of Magnet Errors in the ILC 14 mrad Extraction Line ${ }^{\S \S}$
}

\author{
Dragan Toprek \\ VINCA Institute of Nuclear Sciences, 11001 Belgrade, Serbia*** \\ The Cockcroft Institute, University of Manchester, UK ${ }^{\dagger+\dagger}$ \\ Yuri Nosochkov \\ SLAC National Accelerator Laboratory, Menlo Park, CA 94025, USA
}

\begin{abstract}
The ILC baseline extraction line is designed for 14 mrad horizontal crossing angle between e+ and e- colliding beams at Interaction Point (IP). The extraction optics in the Interaction Region (IR) includes a detector integrated dipole field (anti-DID) to reduce orbit perturbation caused by the detector solenoid and minimize detector background. This paper presents a study of random field and alignment errors in the extraction magnets, compensation of the induced orbit perturbation, and effects of errors on extraction beam power loss. The results are obtained for the baseline ILC energy of $500 \mathrm{GeV}$ center-of-mass and three options of beam parameters.
\end{abstract}

\section{INTRODUCTION}

The ILC baseline extraction line is designed for $14 \mathrm{mrad}$ horizontal crossing angle between $\mathrm{e}^{+}$and $\mathrm{e}-$ colliding beams at Interaction Point (IP) [1]. The extraction optics provides large beam acceptance in order to minimize beam losses caused by large angular spread and long energy tail in the disrupted primary and secondary beams. It also includes dedicated vertical chicanes for beam energy measurement and gamma calorimeter (GAMCAL) diagnostics, and a low $\beta$ focal point with $2 \mathrm{~cm}$ vertical dispersion used as a Compton Interaction Point (CIP) for polarization measurement.

The crossing angle scheme naturally results in a horizontal angle between beam trajectory and direction of the detector solenoid field $B_{\mathrm{s}}$, equal to half-crossing angle $\theta_{\mathrm{c}}=7 \mathrm{mrad}$. This angle creates a systematic horizontal field component $B_{x}=B_{S} \sin \theta_{c}$ on the beam trajectory which causes vertical beam deflection and therefore produces vertical orbit and dispersion, synchrotron radiation and rotation of beam polarization vector. In addition, the solenoid field creates a weak focusing effect in $x-y$ planes and coupling of $x-y$ betatron motion which alter the downstream beam properties. Perturbation of the extracted beam caused by the solenoid needs to be compensated in order to avoid a higher beam loss and preserve the desired beam properties at the Compton IP for polarization measurement. The incoming and extraction optics share a detector integrated dipole field (anti-DID) near the IP in order to reduce the amplitude of the extraction particle trajectories induced by the solenoid and therefore minimize the detector background.

This paper presents calculations of the effects of random field and alignment errors in the extraction magnets with and without their correction, using MAD [2] and DIMAD [3] codes. It is assumed that the incoming beam at IP has the design parameters except an uncorrected $50 \mu$ rad vertical orbit angle generated by the upstream part of the solenoid and anti-DID. The calculations are done for the push-pull version of the extraction optics [4,5] with the final focus drift of

\footnotetext{
$\S \S$ Work supported by the U.S. Department of Energy contract DE-AC02-76SF00515 and by the Cockcroft Institute, University of Manchester, UK.

**** Author's present address.

${ }^{+\dagger \dagger}$ Author's affiliation when this study began.
} 
$L^{*}=3.51 \mathrm{~m}$ and the SiD [6,7] detector solenoid field model, for the nominal ILC energy of $500 \mathrm{GeV}$ center-of-mass (CM) and three options of beam parameters.

\subsection{Extraction Line Optics}

Beam optics of the 14 mrad extraction line and three options of ILC beam parameters are described in Refs. $[4,8]$. Length of the extraction line from IP to dump is about $300 \mathrm{~m}$. The first superconducting (SC) incoming quadrupole QD0 is located at distance of $L^{*}=3.51 \mathrm{~m}$ from IP, and the first extraction SC quadrupole QDEX1 is at $5.5 \mathrm{~m}$ to provide sufficient horizontal separation from the incoming line. These two quadrupoles and the incoming sextupole SD0 will be part of a detector in a push-pull configuration [5]. Beam apertures of the extraction SC quadrupoles QDEX1 and QFEX2A are set to the maximum values of $R=15 \mathrm{~mm}$ and $30 \mathrm{~mm}$, respectively, limited by the separation from the incoming magnets. The remaining downstream extraction quadrupoles are warm magnets starting at distance of 17.19 $\mathrm{m}$ from IP. The extraction quadrupole system is designed to provide: 1 ) a low $\beta$ focal point at $148.6 \mathrm{~m}$ from IP, where the Compton IP will be located, 2) the optimal transformation term $R_{22}=-0.5$ from IP to CIP for efficient polarization measurement, and 3) large chromatic and geometric acceptance for keeping the disrupted beam loss at acceptable level. Downstream of the quadrupoles, the extraction line includes a four bend vertical chicane for measurements of beam energy, a four bend vertical chicane for polarization measurement, and two vertical bends for gamma calorimeter luminosity diagnostics [9]. The polarimeter bends create $2 \mathrm{~cm}$ vertical dispersion at the Compton IP located at center of the polarimeter chicane. After the last GAMCAL bend magnet, the extraction line contains a set of 5 horizontal and 5 vertical fast kickers, located on average $\sim 85 \mathrm{~m}$ before the dump. The rapidly oscillating kicker field $(\sim 1 \mathrm{kHz})$ will sweep the beam along a $R=3 \mathrm{~cm}$ circle at the dump in order to increase the effective beam area for protecting the dump window from high power density of small undisrupted beam and preventing water boiling in the dump vessel.

The extraction collimation system includes two collimators in the chicanes. The first one is placed at center of the energy chicane, $60.7 \mathrm{~m}$ after IP, where momentum dispersion is $17 \mathrm{~mm}$. Its $40 \mathrm{~mm}$ vertical aperture on the low energy side of the beam is set to remove the low energy tail electrons with relative energies below $30-35 \%$. The second collimator at $160.9 \mathrm{~m}$ is inside the polarimeter chicane to protect the Cherenkov detector (at $175.6 \mathrm{~m}$ ) from synchrotron radiation created in the energy chicane bends. Three more collimators (COLW1, COLW2 and COLW3) are included in the final $100 \mathrm{~m}$ section before the dump in order to protect the fast sweeping kickers and limit beam size to within $R=$ $15 \mathrm{~cm}$ at the dump window.

\subsection{Parameter Options and Disrupted Beam Properties at IP}

Distributions of primary disrupted beams at IP were generated using GUINEA-PIG beam-beam simulation code [10]. Beam disruption in collision creates a very long tail of low energy particles and large angular divergence in the outgoing beams. Three beam parameter options were studied [11,12]: 1) nominal (C11), 2) with a large vertical emittance (C13), and 3) with a low beam power (C14). These parameter settings are based on different values of emittance, beam current and beam size at IP, but they provide the same design luminosity. Consequently, the effect of beam disruption varies in these options. The low power option C14 yields the strongest disruption effect leading to a larger extracted beam. The disruption can be further enhanced by a vertical offset between the e+ and e- beams at IP. Therefore, for each parameter option two cases were studied: without a vertical offset and with a vertical offset 
corresponding to maximum disruption effect. The selected full beam-to-beam vertical offsets $\Delta y$ in the C11, C13, C14 options are $200 \mathrm{~nm}, 300 \mathrm{~nm}$, and $120 \mathrm{~nm}$, respectively. Note that in the machine operation any IP offsets will be actively compensated to minimize their effects on luminosity and beam disruption. A horizontal beam offset is not studied in this paper since it reduces the disruption effect for flat beams [13] and therefore is not a concern for extracted beam. Beam parameters used in this study are listed in Table 1 [11].

Table 1: Beam parameters at IP for $500 \mathrm{GeV}$ CM energy.

\begin{tabular}{|l|c|c|c|}
\hline Parameter & $\begin{array}{c}\text { Nominal } \\
\text { C11 }\end{array}$ & $\begin{array}{c}\text { Large Y-Emit } \\
\text { C13 }\end{array}$ & $\begin{array}{c}\text { Low Power } \\
\text { C14 }\end{array}$ \\
\hline Number of particles per bunch, $N$ & $2 \cdot 10^{10}$ & $2 \cdot 10^{10}$ & $2 \cdot 10^{10}$ \\
\hline Number of bunches per train, $N_{b}$ & 2820 & 2820 & 1330 \\
\hline Repetition frequency, $f[\mathrm{~Hz}]$ & 5 & 5 & 5 \\
\hline Norm. emittance, $\gamma \varepsilon_{\mathrm{x}}[\mathrm{mrad}]$ & $1 \cdot 10^{-5}$ & $1.2 \cdot 10^{-5}$ & $1 \cdot 10^{-5}$ \\
\hline Norm. emittance, $\gamma \varepsilon_{\mathrm{y}}[\mathrm{mrad}]$ & $4 \cdot 10^{-8}$ & $8 \cdot 10^{-8}$ & $3.5 \cdot 10^{-8}$ \\
\hline Beta function, $\beta_{\mathrm{x}}{ }^{*}[\mathrm{~mm}]$ & 21 & 10 & 10 \\
\hline Beta function, $\beta_{\mathrm{y}}{ }^{*}[\mathrm{~mm}]$ & 0.4 & 0.4 & 0.2 \\
\hline RMS beam size, $\sigma_{\mathrm{x}}{ }^{*}[\mathrm{~nm}]$ & 655 & 495 & 452 \\
\hline RMS beam size, $\sigma_{\mathrm{y}}{ }^{*}[\mathrm{~nm}]$ & 5.7 & 8.1 & 3.8 \\
\hline RMS bunch length, $\sigma_{\mathrm{z}}{ }^{*}[\mu \mathrm{m}]$ & 300 & 500 & 200 \\
\hline Energy loss by beamstrahlung, $\delta_{\mathrm{BS}}$ & 0.022 & 0.024 & 0.057 \\
\hline Beam power, $P[\mathrm{MW}]^{-2}$ & 11.3 & 11.3 & 5.3 \\
\hline Luminosity, $\mathcal{L}\left[\mathrm{cm}^{-2} \mathrm{~s}^{-1}\right]$ & $2 \cdot 10^{34}$ & $2 \cdot 10^{34}$ & $2 \cdot 10^{34}$ \\
\hline
\end{tabular}

Tracking of $\sim 10^{5}$ particles is typically sufficient for adequate simulation of beam distribution in the extraction line and for calculation of relatively high beam loss in collimators. However, accurate estimate of much lower losses in magnets typically requires a much higher statistics of $>10^{7}$ particles in the simulated beam. The latter, unfortunately, significantly increases the simulation running time and requires large computer storage space. Fortunately, for the purpose of beam loss calculation in magnets, it is not necessary to track a full beam because only very low energy particles are lost in extraction magnets due to over-focusing and dispersion. In this case, it is sufficient to track only the lowest part of beam energy tail and particles with large initial $x-y$ angles. As a result, two types of particle data files were used in this study. The low statistics files containing total of $\sim 7 \cdot 10^{4}$ particles in two beams were used for evaluation of beam distribution and polarization at Compton IP, and for calculation of high beam loss in extraction collimators. The high statistics files with total of up to $3.5 \cdot 10^{7}$ particles in two beams were generated for the purpose of beam loss calculation in extraction magnets. But for tracking simulations, these files were reduced to much smaller files containing $\leq 10^{5}$ particles with relative energies below 65\% and/or initial $x-y$ angles larger than \pm 0.5 mrad. For maximum statistics, the above files contain both $\mathrm{e}^{+}$and $\mathrm{e}-$ colliding beam distributions, but assuming the same charge 
for all particles. In case of ideal collisions without beam offset at IP, the e+ and e- disrupted distributions are identical, therefore combining the two beams does not make difference. In case of a non-zero IP vertical offset, the outgoing e+ and e- beams will have the same but opposite systematic offsets of $y$ and $y^{\prime}$ at IP. In this case, combining e+ and ebeams is equivalent to combining two beams with initial positive and negative vertical offsets. Therefore, the resultant power loss will be an average of power losses with positive and negative offsets at IP.

The disrupted beam distributions at IP, obtained by GUINEA-PIG, for the three beam parameter options without and with vertical offset are shown in Ref. [8] in Figures 4-9, and distributions of particle energies are presented in Fig. 10 in the same Reference.

Fig. 1 shows the disrupted $\beta$ functions and dispersion in the 14 mrad extraction line without magnet errors, as calculated by MAD, for the Nominal parameter option C11 in presence of the detector solenoid field, the anti-DID field and orbit correction in the solenoid and polarimeter chicane regions. The incoming vertical orbit angle at IP is assumed to be $50 \mu \mathrm{rad}$. Beta functions are focused by the extraction quadrupoles to a low $\beta$ waist at the Compton IP ( $s=148.6$ $\mathrm{m})$ with the transformation matrix term $R_{22}=-0.5$ from IP to CIP, suitable for polarization measurements. The orbit caused by the post-IP detector solenoid, anti-DID field and $50 \mu$ rad IP y-angle is canceled locally in the solenoid and polarimeter regions as shown Fig. 2. At the Compton IP the local polarimeter correctors are set to make the same orbit angle as at the IP, i.e. $y^{\prime}(C I P)=50 \mu$ rad and $x^{\prime}(C I P)=0$. Although the local solenoid correctors are not specifically designed to compensate the solenoid dispersion, they provide almost complete correction as shown in Fig. 1, because their positions are close to the solenoid. This compensation should be sufficient for polarization measurement.

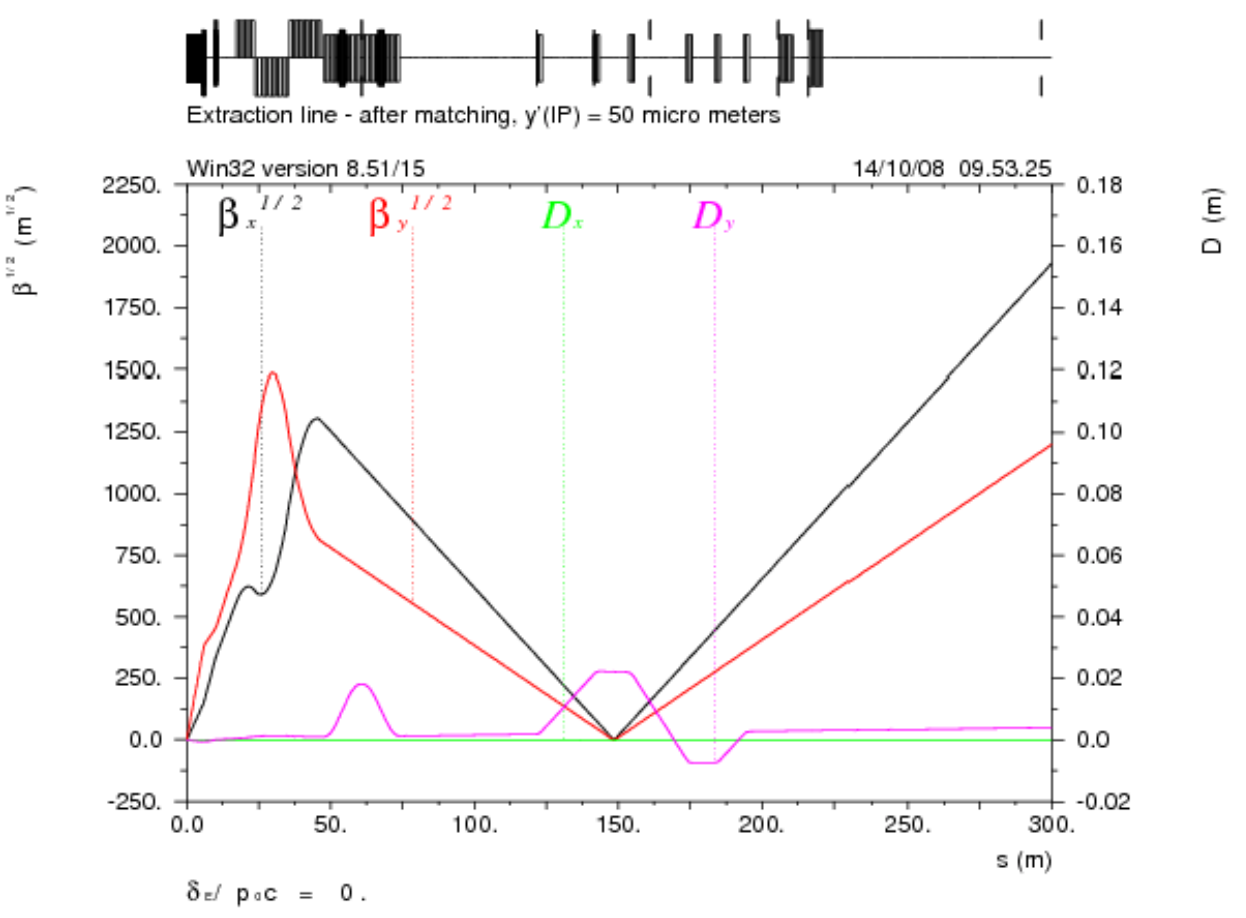

Figure 1: Disrupted $\beta$ functions and dispersion in the $14 \mathrm{mrad}$ extraction line for the Nominal parameter option. The IP vertical orbit angle is $y^{\prime}(I P)=50 \mu \mathrm{rad}$, and the Compton IP angle is corrected to $y^{\prime}(C I P)=50 \mu \mathrm{rad}$. 
The solenoid weak focusing creates a shift of the low beta waist away from Compton IP resulting in a larger beam size at the CIP. This effect can be compensated by using two quadrupole corrector coils on the extraction quadrupoles. These correctors can be used to maintain the point-to-point focusing from IP to CIP by adjusting the transfer matrix terms $R_{12}$ and $R_{34}$ to zero. Previous studies did not find a significant effect of solenoid coupling on the extraction beam properties; therefore no coupling correction has been considered.

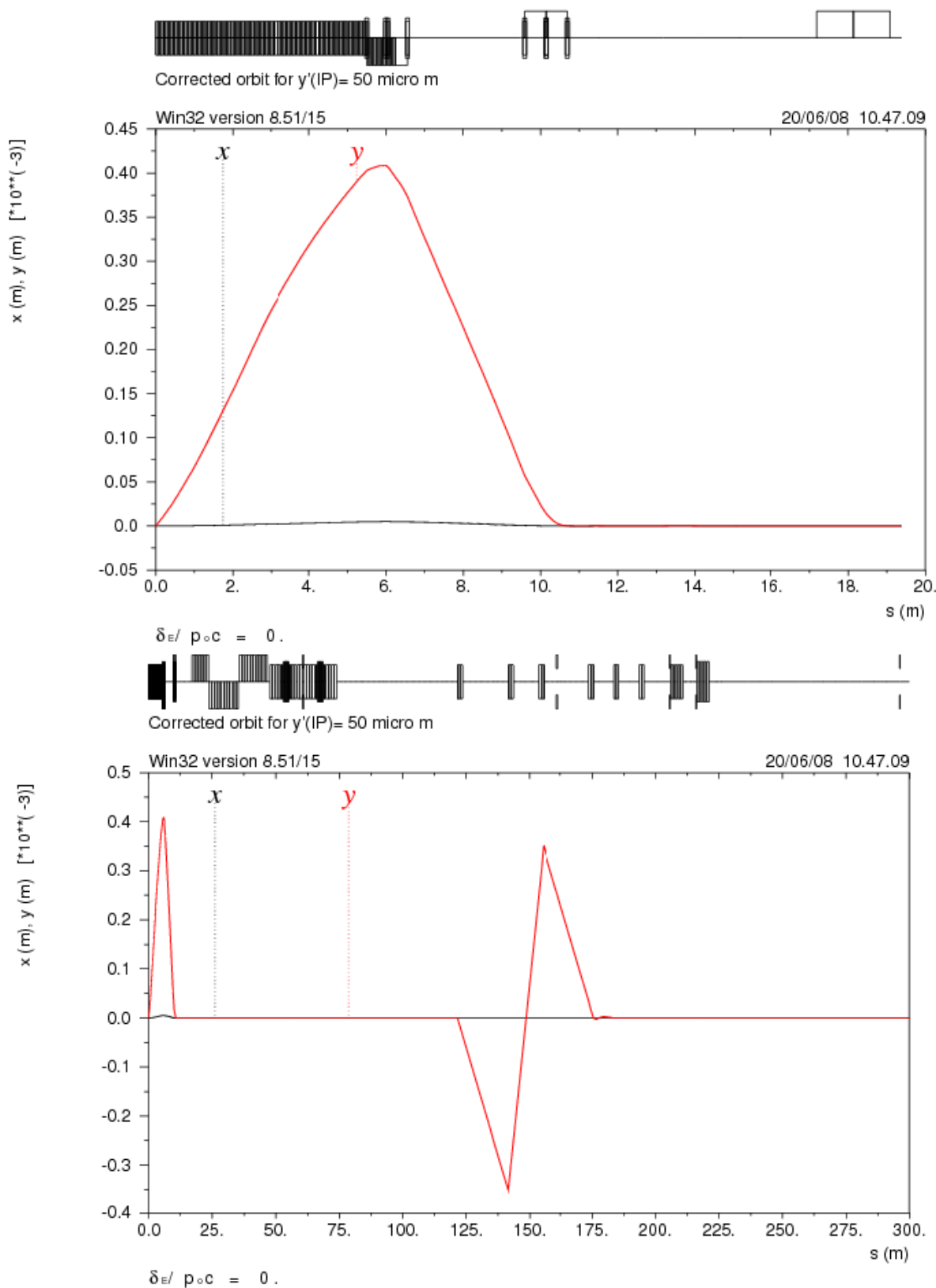

Figure 2: Corrected $X$ and $Y$ orbit in the $14 \mathrm{mrad}$ extraction line. The top figure shows the first $20 \mathrm{~m}$ after IP. The IP vertical orbit angle is $y^{\prime}(I P)=50 \mu \mathrm{rad}$, and the Compton IP angle is corrected to $y^{\prime}(C I P)=50 \mu \mathrm{rad}$. 


\section{BEAM POWER LOSS WITHOUT MAGNET ERRORS}

The primary disrupted beam power losses were calculated using DIMAD tracking. As described earlier, two types of particle data files were used. The relatively low statistics files with $\sim 7 \cdot 10^{4}$ particles for combined beams were used to calculate the relatively large losses on extraction collimators. The high statistics files based on $3.5^{1} 10^{7}$ particles in the combined beams were used to estimate low losses in the extraction magnets. Since it is known that only particles with the lowest energies and largest IP angles are lost in the magnets, the high statistics files were reduced to much smaller files containing only beam tails where the relative particle energies are below $65 \%$ and/or their IP angles exceed 0.5 mrad. In this tracking, the solenoid, the anti-DID and the compensating dipole correctors are turned on, and the vertical orbit at Compton IP is adjusted to have the same angle as at IP, i.e. $y^{\prime}(C I P)=y^{\prime}(I P)=50 \mu \mathrm{rad}$.

The tracking found no power losses in the extraction quadrupoles for the Nominal and Low Y-emittance parameter options. Therefore only the results for the Low Power option are presented in Table 2. Note that no primary loss was observed in the sensitive SC quadrupoles, and the maximum loss in warm quadrupoles is $1.4 \mathrm{~W}$ for collisions without IP offset and $25 \mathrm{~W}$ for collisions with IP $y$-offset. This loss level is acceptable and should not cause long-term radiation damage. Note that the large beam offsets at IP will be actively corrected in real beam operation in order to maintain high luminosity, therefore the high beam loss caused by the IP offsets will be limited to short periods of time and therefore on average will be significantly lower.

In Table 2 the numbers shown in black colour correspond to the case when the field and alignment errors are NOT applied. The meaning of the other colours (red and green) will be explained later (see Section 4). The columns "BEFORE CORRECTIONS" and "AFTER CORRECTIONS" in Table 2 correspond to the cases with errors before and after correction, respectively. The description of errors and their type will be also explained in Section 4.

Power losses in the extraction collimators, in the case when the field and alignment errors are not applied, are summarized in Table 3 for the three parameter options without and with IP offset. In this tracking, the fast sweeping kickers were set to sweep the beam on $R=3 \mathrm{~cm}$ circle at dump. Under ideal IP conditions without beam offset, the maximum power loss in a single collimator is near $21 \mathrm{~kW}$. With the large $y$-offset, the maximum collimator loss can be up to $64 \mathrm{~kW}$. However, as mentioned earlier, the IP offsets will be actively corrected and therefore the average loss will be lower. Most of the high loss occurs in the final three collimators COLW1,2,3 which purpose is to clean-up the beam tails before the dump. Since there are no magnets or diagnostic in most of this region, this high loss is not of a significant concern. The clean-up collimator COLE and protection collimator COLCD are located in the energy and polarimeter chicanes where the beam loss is much lower. 
Table 2: Primary disrupted beam loss (W) in the extraction quadrupoles for the Low Power parameter option without and with IP $y$-offset for the selected magnet errors. Black colour - errors are not applied, green - bend magnet field errors (in brackets - bend magnet misalignment), red - quad field errors (in brackets - quad misalignment), blue - detector solenoid field error (in brackets - solenoid misalignment).

\begin{tabular}{|l|c|c|c|c|}
\cline { 2 - 5 } \multicolumn{1}{c|}{} & \multicolumn{2}{c|}{ BEFORE CORRCTIONS } & \multicolumn{2}{c|}{ AFTER CORRECTIONS } \\
\hline Quadrupole & C14 & C14, y-offset & C14 & C14, y-offset \\
\hline QDEX1 (SC) & 0 & 0 & 0 & 0 \\
\hline QFEX2A (SC) & 0 & 0 & 0 & 0 \\
\hline QFEX2B & 0 & 0 & 0 & 0 \\
\hline QFEX2C & 0 & 0 & 0 & 0 \\
\hline QFEX2D & 0 & 0 & 0 & 0 \\
\hline QDEX3A & 0 & 0.3 & 0 & 0.3 \\
& $0(0)$ & $0.7(0.7)$ & $0(0)$ & $0.7(0.7)$ \\
& $0(0)$ & $0.7(0.7)$ & $0(0)$ & $0.7(0.7)$ \\
& $0(0)$ & $0.7(0.7)$ & $0(0)$ & $0.9(0.9)$ \\
\hline QDEX3B & 0 & 0 & 0 & 0 \\
& $0(0)$ & $0(0)$ & $0(0)$ & $0(0)$ \\
& $0(0)$ & $0(0.2)$ & $0(0)$ & $0(0.2)$ \\
& $0(0)$ & $0(0)$ & $0(0)$ & $0(0.1)$ \\
\hline QDEX3C & 0.1 & 0 & 0.1 & 0 \\
& $0.2(0.2)$ & $0(0)$ & $0.2(0.2)$ & $0(0)$ \\
& $0.2(0.2)$ & $0(0)$ & $0.2(0.2)$ & $0(0)$ \\
& $0.2(0.2)$ & $0(0)$ & $0.2(0.2)$ & $0(0)$ \\
\hline QDEX3D & 0.5 & 0 & 0.5 & 0 \\
& $1.1(1.1)$ & $0(0)$ & $1.0(1.0)$ & $0(0)$ \\
& $1.1(1.3)$ & $0(0)$ & $1.0(1.0)$ & $0(0)$ \\
& $1.1(1.3)$ & $0(0)$ & $1.0(1.3)$ & $0(0)$ \\
\hline QDEX3E & 1.0 & 0 & 1.0 & 0 \\
& $2.1(2.1)$ & $0(0)$ & $2.1(2.1)$ & $0(0)$ \\
& $2.0(2.0)$ & $0(0)$ & $2.0(2.0)$ & $0(0)$ \\
& $2.1(2.1)$ & $0(0)$ & $2.1(2.1)$ & $0(0)$ \\
\hline QFEX4A & 1.4 & 0.1 & 1.4 & 0.1 \\
& $2.8(2.8)$ & $0.3(0.3)$ & $2.8(2.4)$ & $0.2(0.2)$ \\
& $2.8(2.6)$ & $0.2(0.2)$ & $2.8(2.8)$ & $0.2(0.2)$ \\
& $2.8(2.6)$ & $0.2(0.2)$ & $2.9(2.9)$ & $0.2(0.5)$ \\
\hline QFEX4B & 0 & 0 & 0 & 0 \\
\hline QFEX4C & 0.4 & 0.4 & 0.4 & 0.4 \\
& $0.7(0.7)$ & $0.7(0.7)$ & $0.9(0.4)$ & $0.7(0.3)$ \\
& $0.7(0.7)$ & $0.7(0.7)$ & $0.5(0.7)$ & $0.4(0.4)$ \\
& $0.7(0.7)$ & $0.7(0.7)$ & $0.9(0.4)$ & $0.7(0.5)$ \\
\hline & 0.3 & 0.3 & 0.3 & 0.3 \\
& $0.6(0.6)$ & $0.5(0.5)$ & $0.4(0.6)$ & $0.5(0.2)$ \\
& $0.6(0.6)$ & $0.5(0.2)$ & $0.6(0.8)$ & $0.5(0.5)$ \\
& $0.6(0.9)$ & $0.5(0.5)$ & $0.6(0.6)$ & $0.5(0.5)$ \\
\hline & 0.1 & 25.4 & 0.1 & 25.4 \\
& $0.2)$ & $51.8(51.8)$ & $1.2(0.2)$ & $77.0(76.8)$ \\
& $50.8(25.8)$ & $0.2(0.2)$ & $49.8(49.8)$ \\
& $51.8(51.8)$ & $0.2(0.2)$ & $76.5(38.4)$ \\
\hline
\end{tabular}


Table 3: Primary disrupted beam loss $(\mathrm{kW})$ in the extraction collimators for the Nominal, Low $\mathrm{Y}$-emittance and Low Power parameter options without and with IP $y$-offset in the case when the errors are NOT applied. Fast sweeping kickers are set to sweep the beam on $R=3 \mathrm{~cm}$ circle at dump.

\begin{tabular}{|l|c|c|c|c|c|}
\hline Parameter Option & COLE & COLCD & COLW1 & COLW2 & COLW3 \\
\hline C11 & 0 & 0 & 0.16 & 0.08 & 0.93 \\
\hline C11, y-offset & 0 & 0.08 & 3.85 & 3.34 & 8.92 \\
\hline C13 & 0 & 0.07 & 1.42 & 1.14 & 5.72 \\
\hline C13, y-offset & 0 & 0 & 2.32 & 1.71 & 8.49 \\
\hline C14 & 0.10 & 0.97 & 10.27 & 8.15 & 21.46 \\
\hline C14, y-offset & 0.34 & 11.53 & 63.66 & 38.60 & 61.34 \\
\hline
\end{tabular}

\section{MAGNET ERRORS AND CORRECTION}

The real magnets will have field and alignment errors causing beam perturbation. In this paper, we consider that the field errors are applied to the detector solenoid, to the quadrupoles and dipoles and have a Gaussian width of $10^{-3}$ relative to the nominal setting and are applied to the elements in an uncorrelated manner. The misalignment is applied in the same way, where the Gaussian width of horizontal and vertical offset is $310 \mu \mathrm{m}$, and the roll error Gaussian width is $1.5 \mathrm{mrad}$. The longitudinal component of the solenoid field error $B_{s, E r r}(s)$ is chosen to be $+0.5 \%$ of the nominal field value $B_{s}(s)$; i.e. the total field is $B_{s, E r r}(s)=1.005 \cdot B_{S}(s)$. Bend field errors are simulated by thin lens kickers placed in the middle of the bend magnets. One random set of errors is used in this study. The rms field and alignment errors are summarized in Table 4.

Table 4: RMS values of field and alignment errors in the solenoid, quadrupoles and bend magnets.

\begin{tabular}{|c|c|c|c|c|c|c|}
\hline ELEMENTS & $\Delta B / B$ & $\begin{array}{c}\Delta x \\
{[\mu \mathrm{m}]}\end{array}$ & $\begin{array}{c}\Delta \theta_{X} \\
{[\mu \mathrm{rad}]}\end{array}$ & $\begin{array}{c}\Delta y \\
{[\mu \mathrm{m}]}\end{array}$ & $\begin{array}{c}\Delta \theta_{Y} \\
{[\mu \mathrm{rad}]}\end{array}$ & $\begin{array}{c}\Delta \theta_{S} \\
{[\mu \mathrm{rad}]}\end{array}$ \\
\hline$\sigma_{\text {SOLEN }}$ & 0.005 & 50 & 500 & 50 & 500 & 0 \\
\hline$\sigma_{\text {QUAD }}$ & 0.001 & 100 & 0 & 50 & 0 & 350 \\
\hline$\sigma_{\text {BEND }}$ & 0.005 & 310 & 0 & 310 & 1500 & 1500 \\
\hline
\end{tabular}

The disrupted beam losses on the normal conducting magnets should be limited to $100 \mathrm{~W} / \mathrm{m}$ to avoid long-term radiation damage. The collimator losses can be much higher depending on the collimator design.

The errors applied to the extraction line result in a perturbation of the linear transport optics and distortion of the beam orbit. These distortions need to be corrected to give good polarimeter performance and ensure the disrupted beam power losses remain acceptable. The orbit correction at the secondary focus (Compton IP) and downstream of the polarimeter chicane can be done using the same set of kickers which are already used to correct the orbit generated by 
the solenoid [8]. These kickers are labeled as VKEX1, VKEX2, HKEX1, HKEX2, VKPOL1, VKPOL2, HKPOL1, HKPOL2 and are located in the solenoid and polarimeter regions [8]. To ensure a good measurement at the polarimeter, the effects of focusing errors at the Compton IP will be compensated using two corrector coils on the extraction quadrupoles to maintain transfer matrix elements $R_{12}$ and $R_{34}$ close to zero between the IP and CIP.

Effects of various magnet errors on extracted beam trajectory are shown in Figures 3-8 (case $a$ - before correction; case $b$ - after correction), as calculated by MAD. Note that misalignment in these figures includes both the x/y magnet offsets and roll errors.

Effects of various errors on energy deposition in collimators for different beam parameter sets are presented in Tables 5-7, where the results after correction are shown in red colour. The effects of errors on energy deposition in quadrupoles for different beam parameter sets are presented in Table 2 shown earlier, where the column labeled “AFTER CORRECTIONS” is for the results after correction of the corresponding errors.

Tables 2 and 5-7 show that the effects of magnets errors do not significantly increase the beam power loss. The orbit correctors cancel the orbit perturbation of the beam core after the solenoid and in the polarimeter chicane, and maintain the correct beam core position and angle at CIP. However they also create residual dispersion which in some cases may somewhat increase losses in the beam low energy tail.

Tables 8-10 show the horizontal $\langle x\rangle$ and vertical $\langle y\rangle$ offsets of the beam core with $d p / p<1 \%$ at the Compton IP, the average spin projection $P[8,14,15]$, and percentage of the beam particles within the laser spot $\kappa$ at the Compton IP for different beam parameter sets (with and without $y$ offset) when various types of errors are applied. The results after corrections are indicated in red colour. Here, the largest orbit offsets at Compton IP are caused by bend field and roll errors and quadrupole misalignment which are compensated by the orbit correctors. In most cases the correctors are able to correct the orbit at CIP to the original level without errors. They also maximize the percentage of particles within the laser spot. Note that the calculated $\langle y\rangle$ offset at CIP has a small systematic negative component due to the design $2 \mathrm{~cm}$ vertical dispersion.

\section{CONCLUSION}

Effects of field and alignment errors in the ILC extraction line magnets were studied using one random set of errors for three ILC beam parameter options with and without beam offset at IP. It is shown that orbit perturbation of the beam core caused mostly by bend field and roll errors, quadrupole and solenoid misalignment and the incoming beam orbit can be corrected locally in the solenoid and polarimeter chicane regions using eight dipole correctors. The polarimeter correctors can also effectively adjust the orbit angle and position at the Compton IP for efficient polarization measurement. It is found that the studied errors do not significantly increase the extraction beam power loss even without orbit correction. The orbit correctors generate additional dispersion which in some cases may somewhat increase losses in the beam low energy tail. The calculated power losses in extraction magnets and collimators are within the acceptable range. 

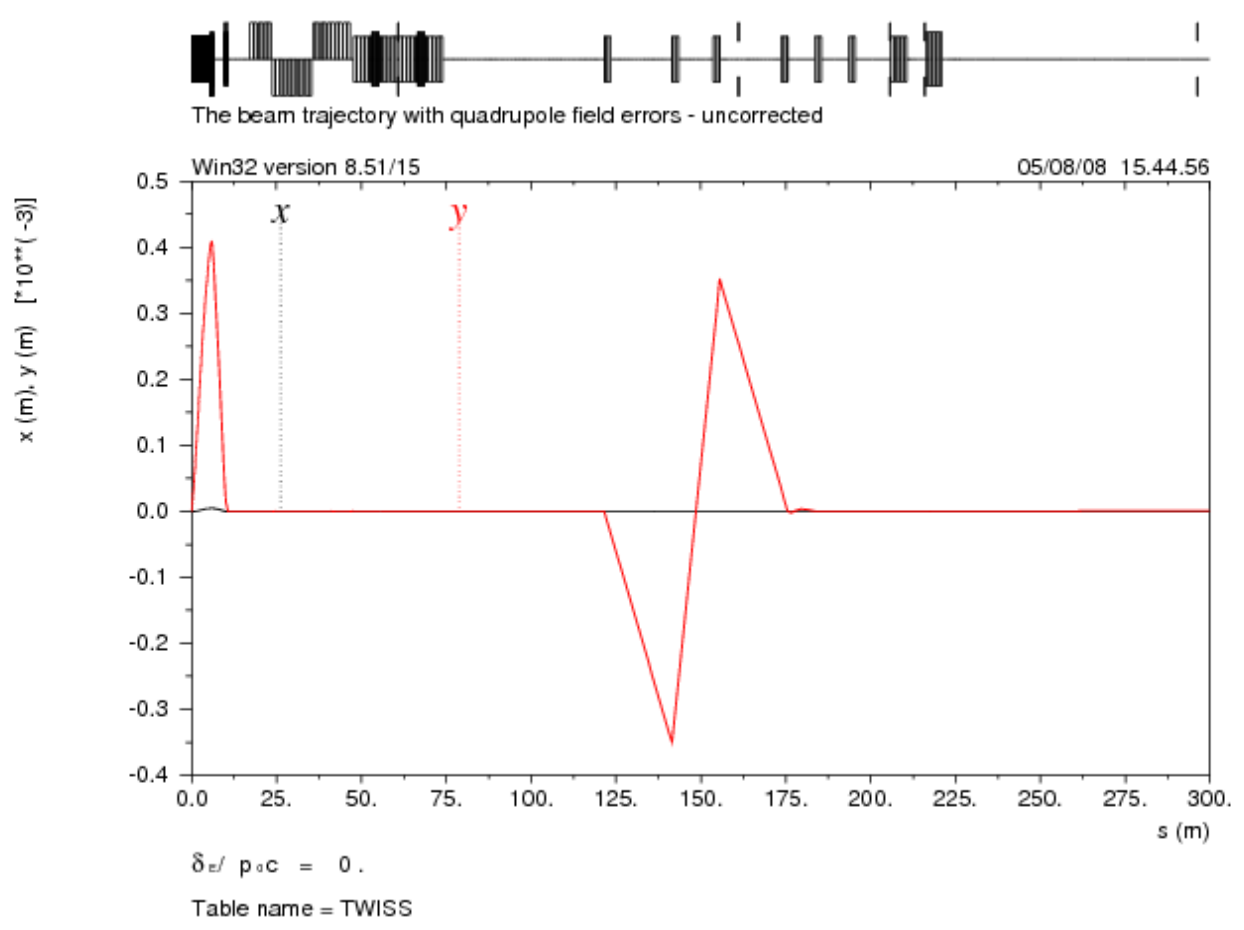

Figure 3a: Beam trajectory with quadrupole field errors before correction.

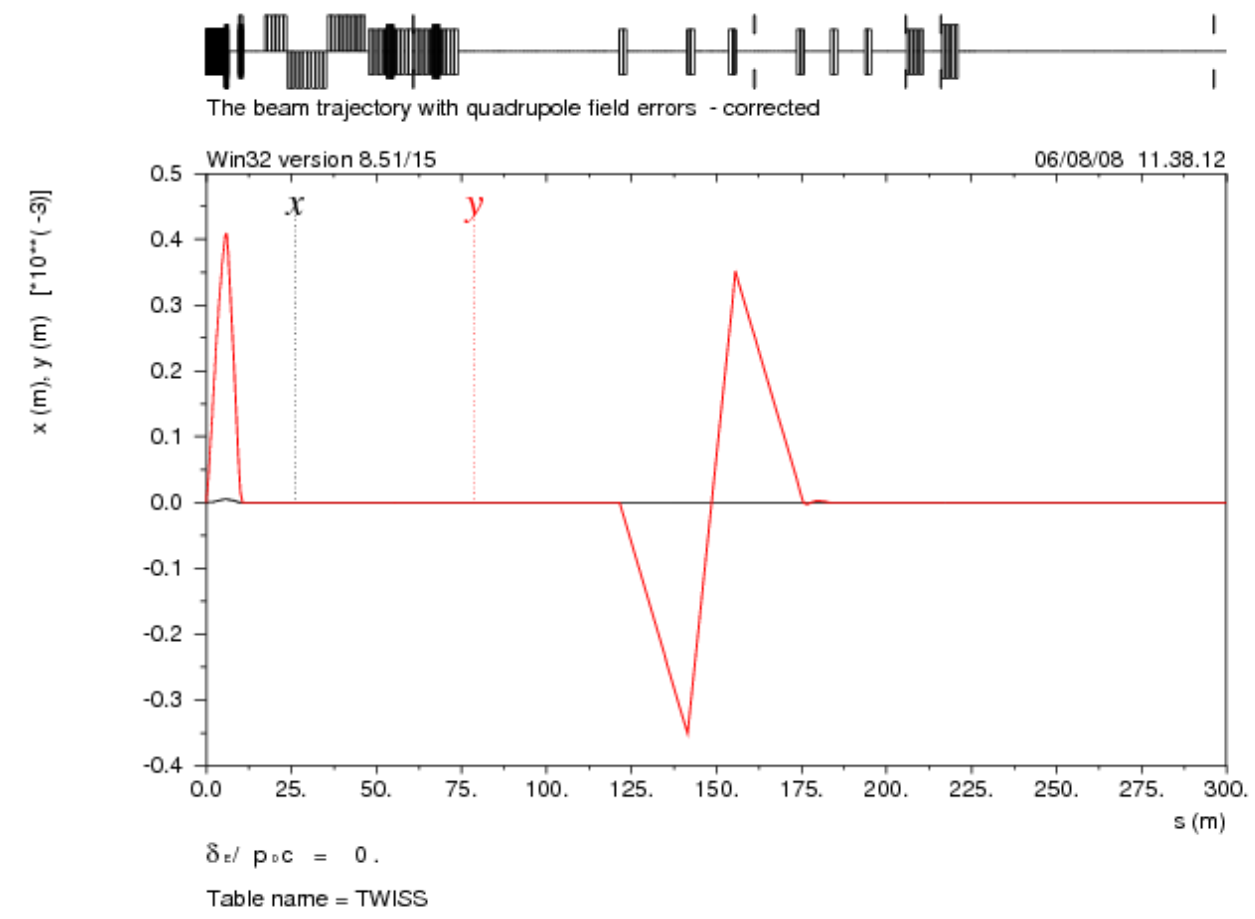

Figure 3b: Beam trajectory with quadrupole field errors after correction. 


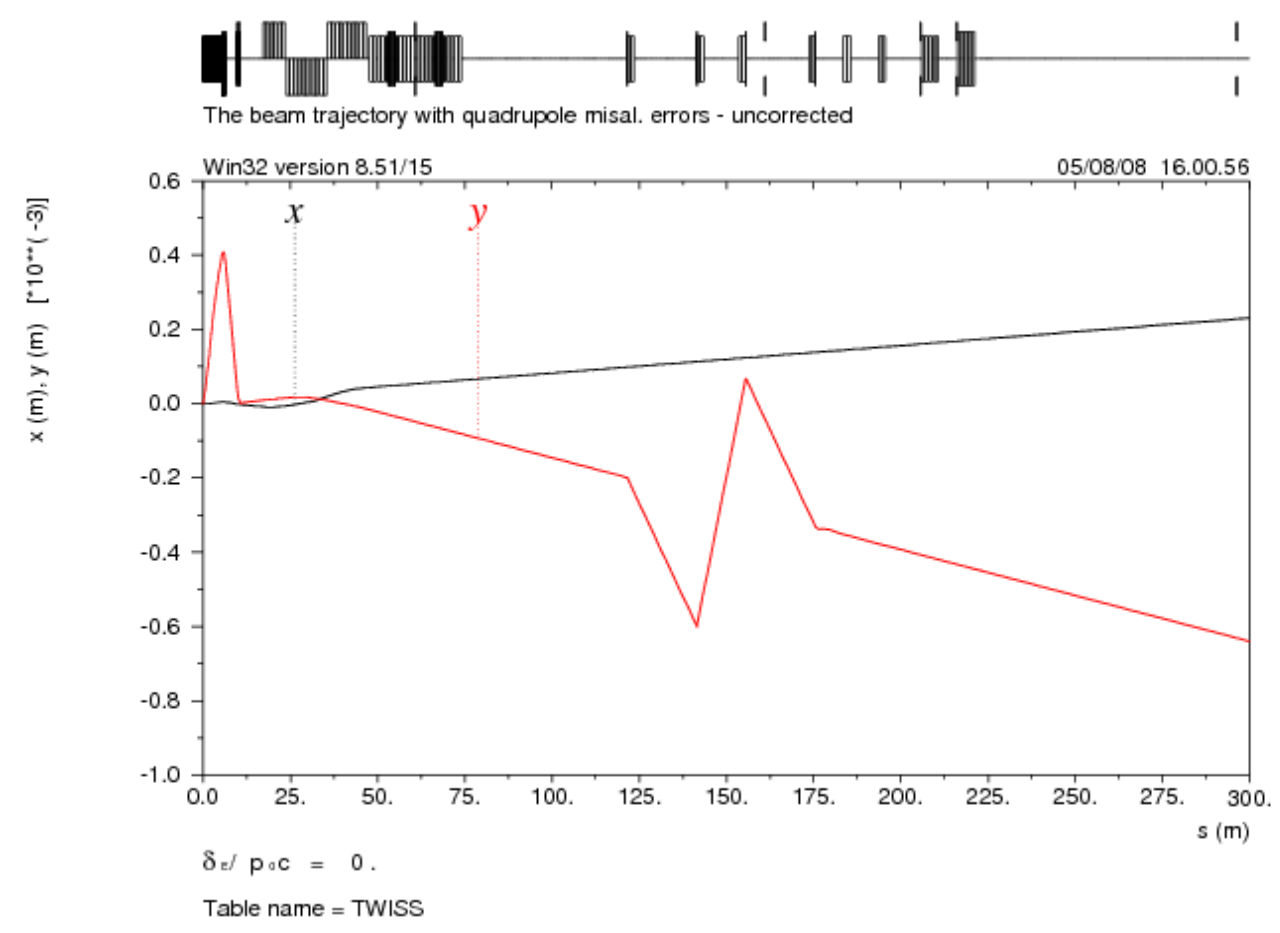

Figure 4a: Beam trajectory with quadrupole alignment errors before correction.

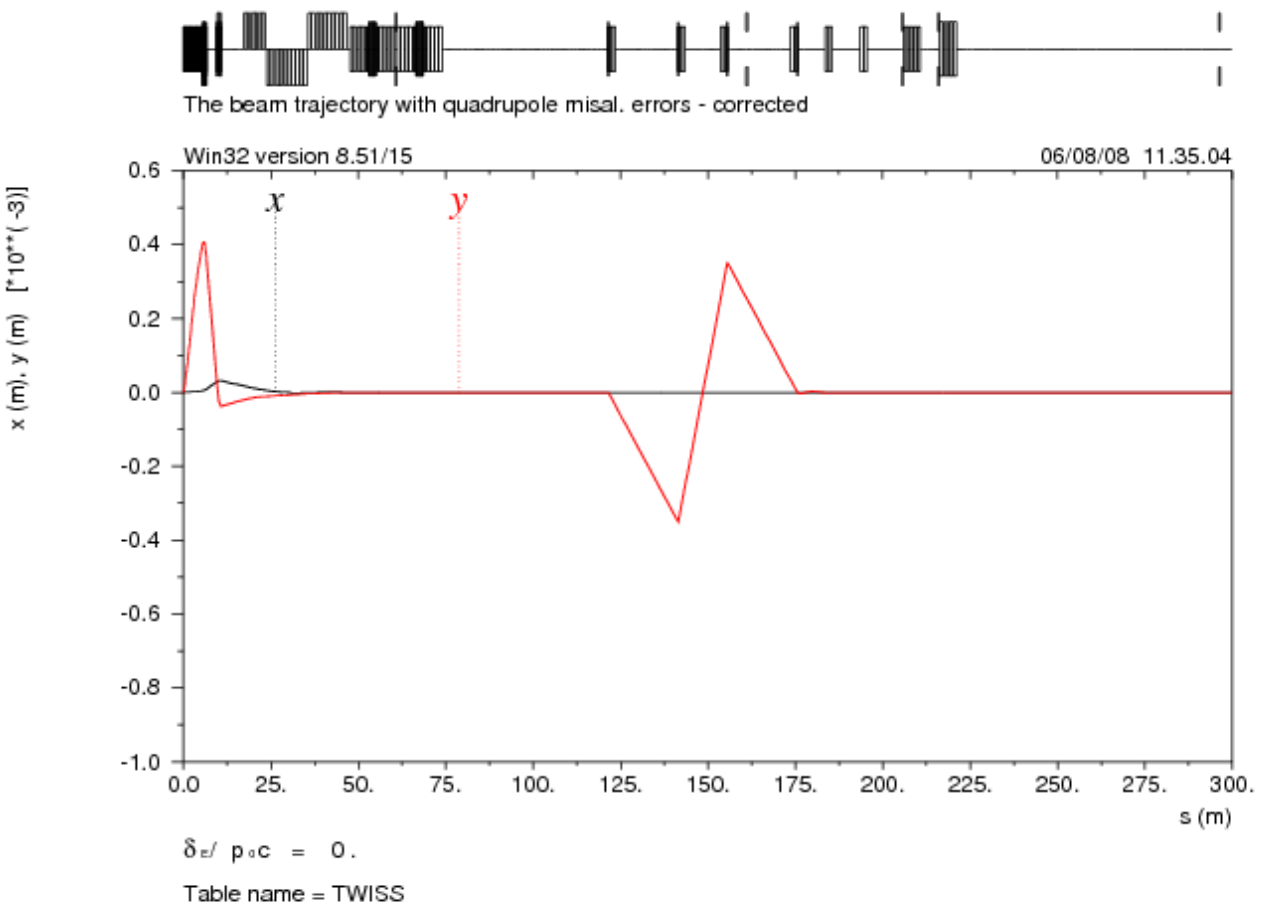

Figure 4b: Beam trajectory with quadrupole alignment errors after correction. 


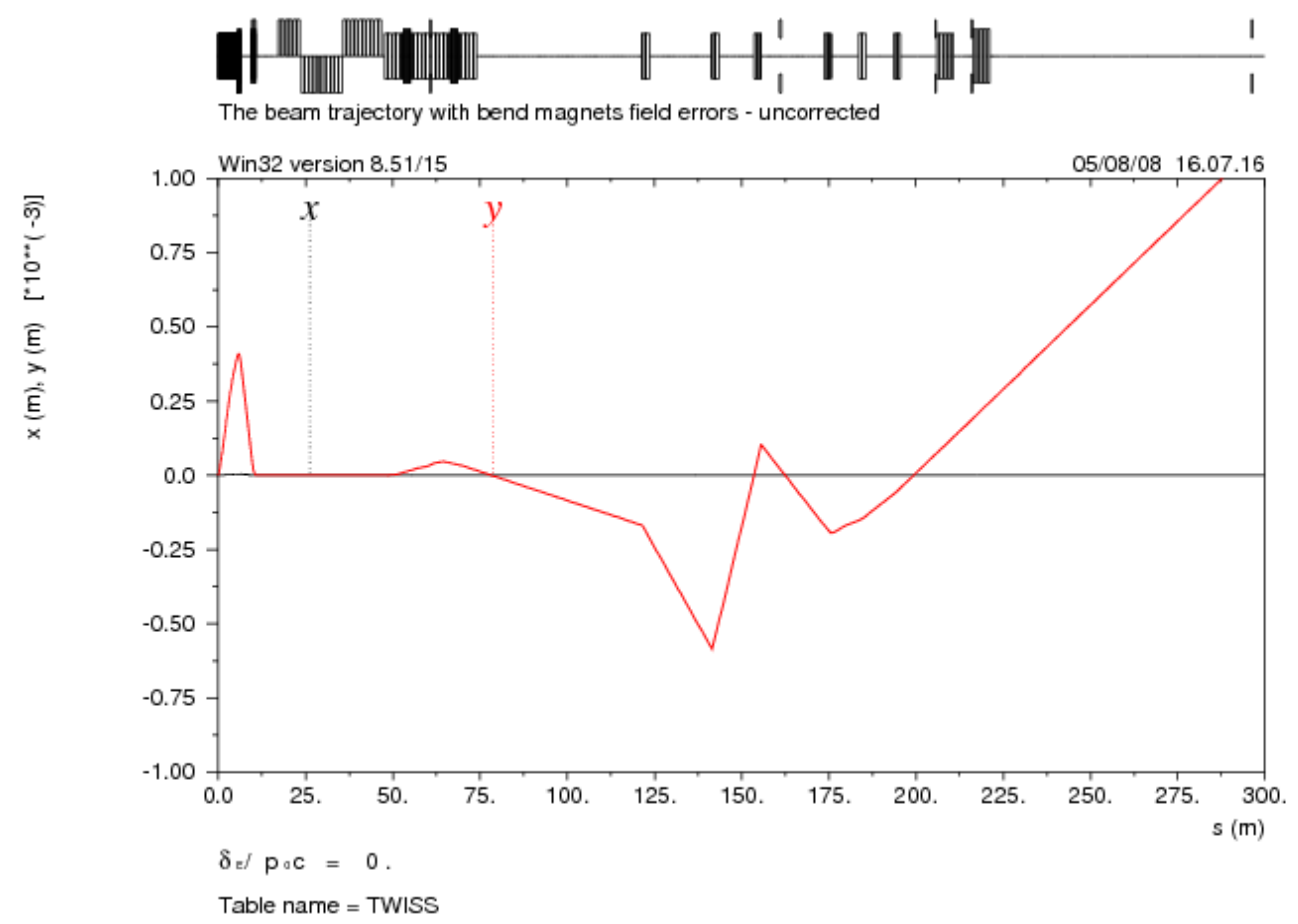

Figure 5a: Beam trajectory with bend magnet field errors before correction.

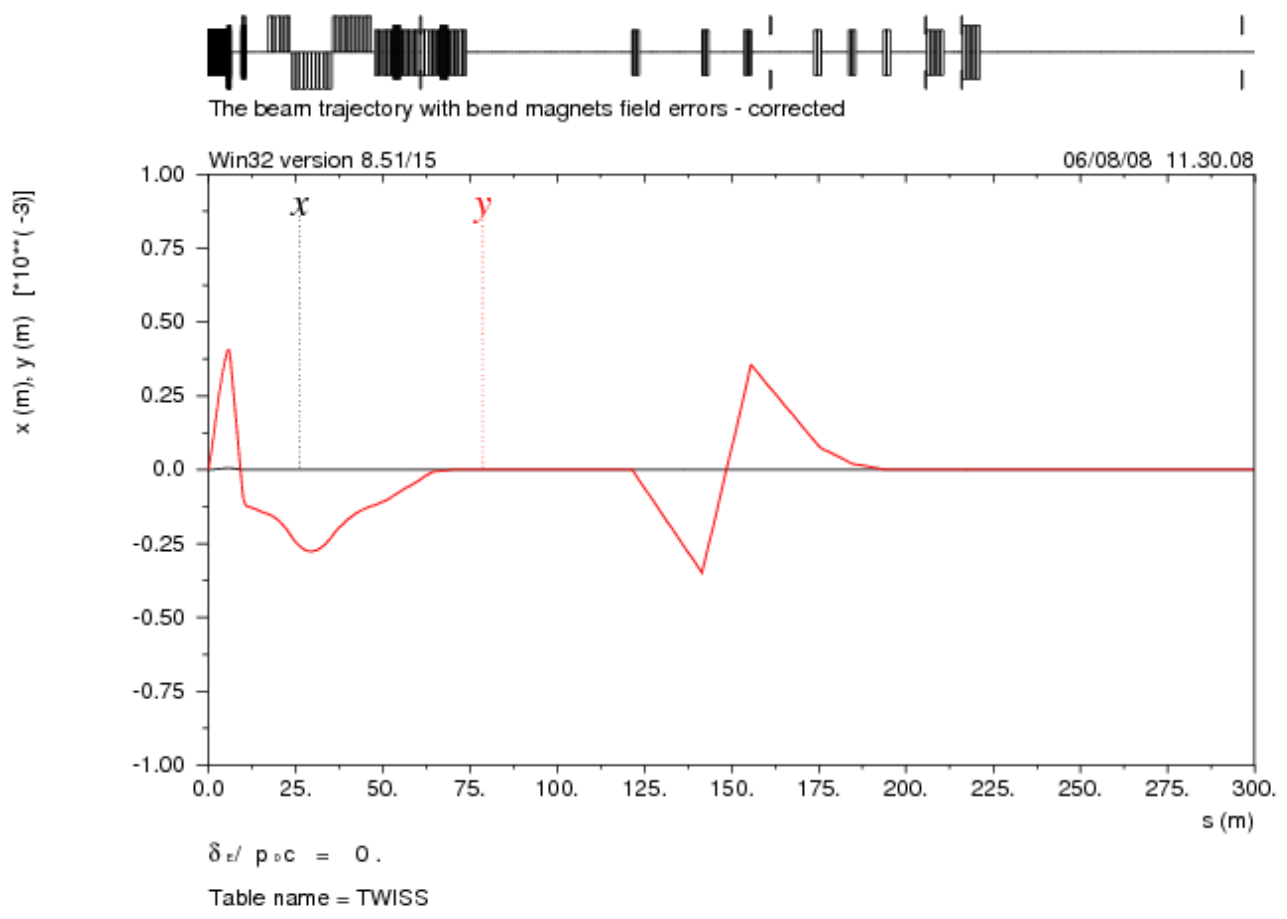

Figure 5b: Beam trajectory with bend magnet field errors after correction. 


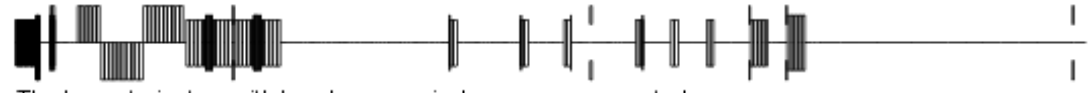

The beam trajectory with bend magn. misal. errors - uncorrected

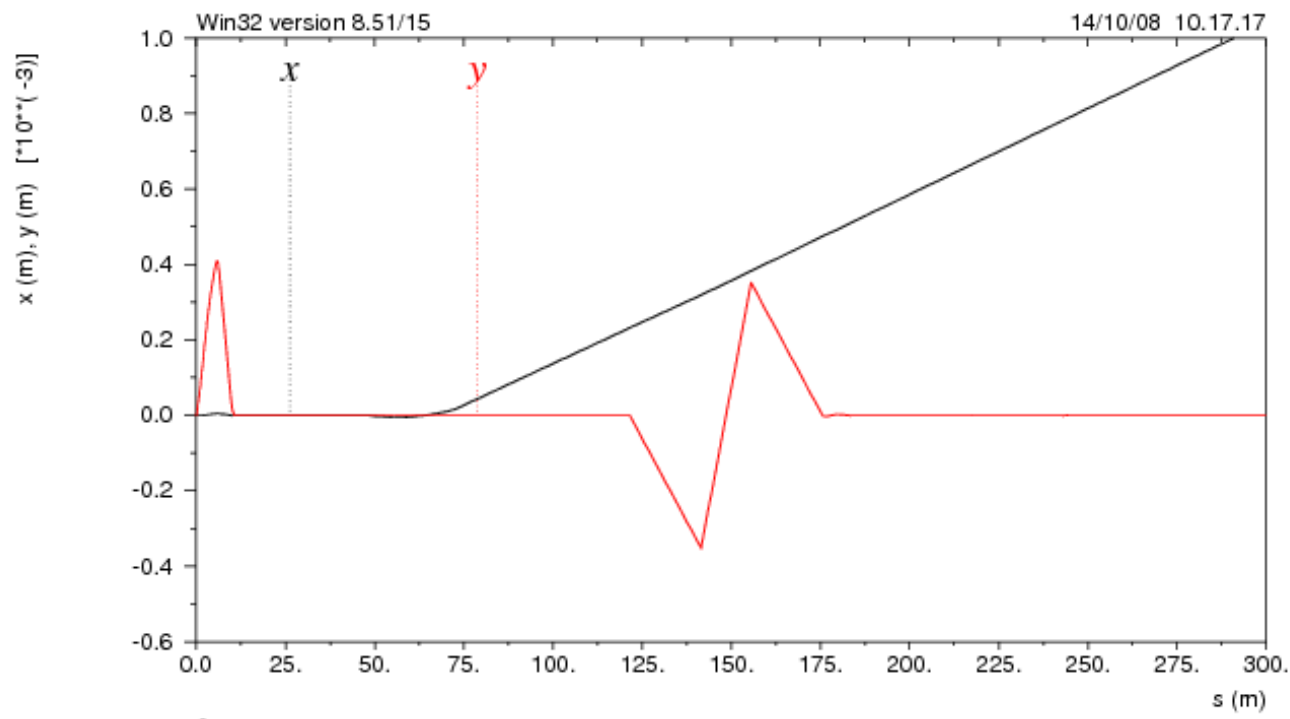

$\delta_{E} / \mathrm{p}_{\circ} \mathrm{C}=0$.

Table name $=$ TWISS

Figure 6a: Beam trajectory with bend magnet alignment errors before correction.

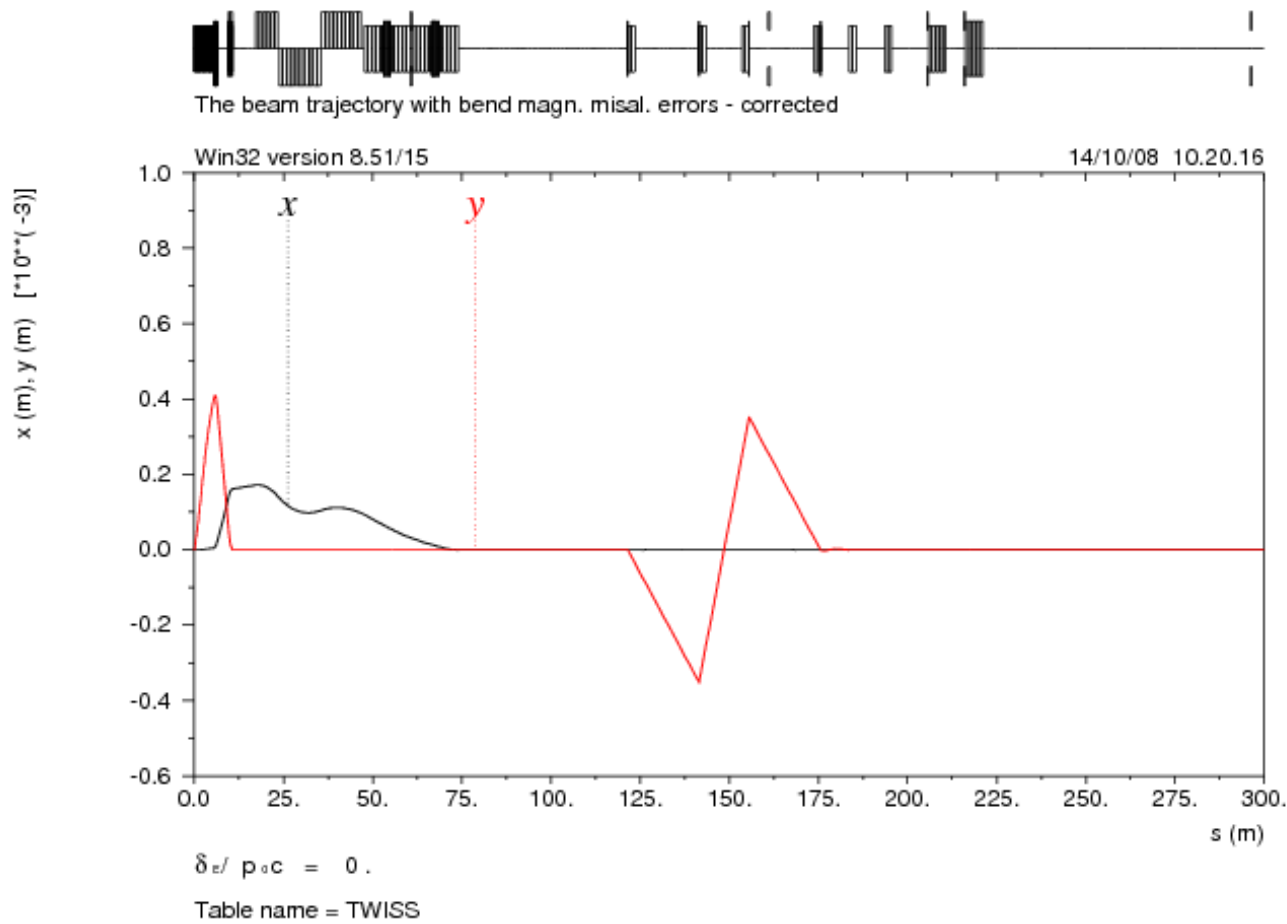

Figure 6b: Beam trajectory with bend magnet alignment errors after correction. 


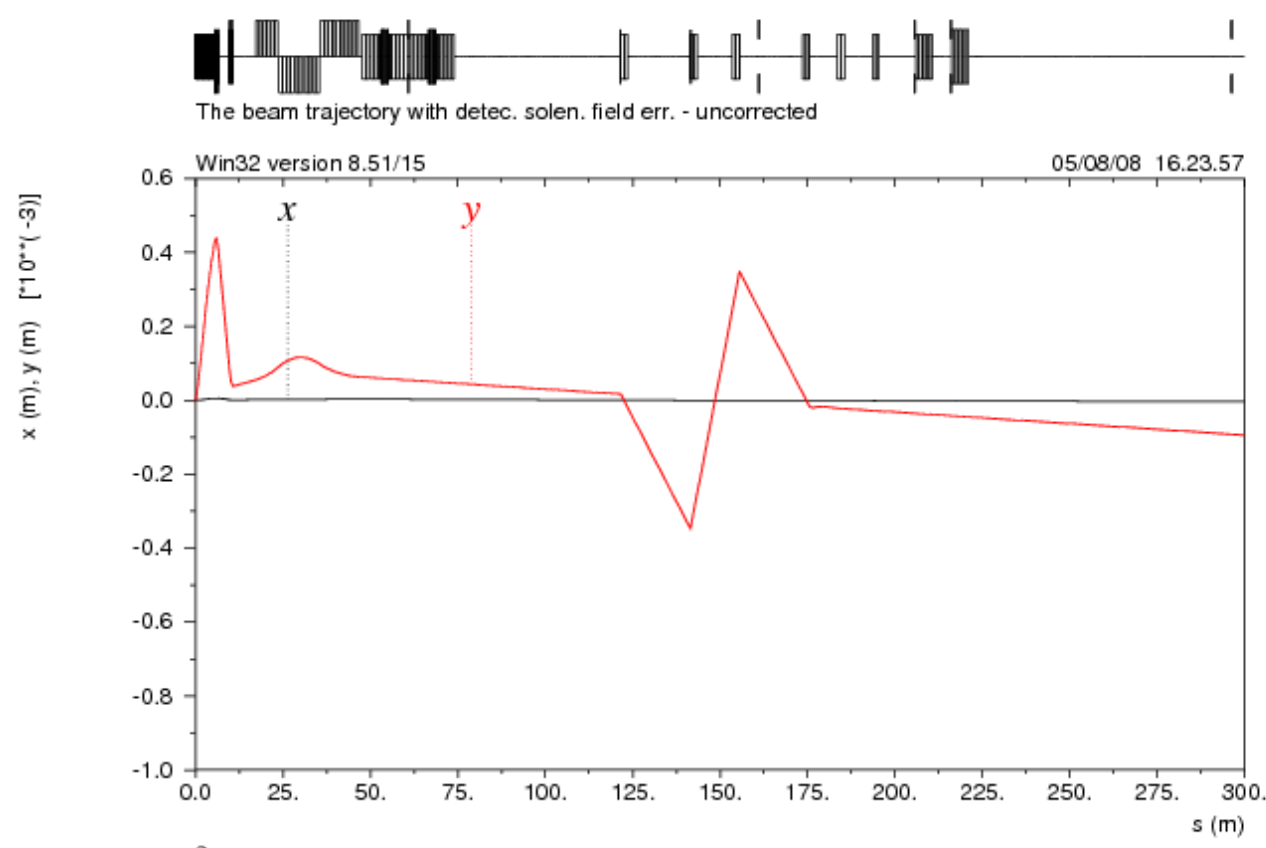

$\delta_{E} / \mathrm{p} \circ \mathrm{c}=0$.

Table name $=$ TWISS

Figure 7a: Beam trajectory with detector solenoid field errors before correction.

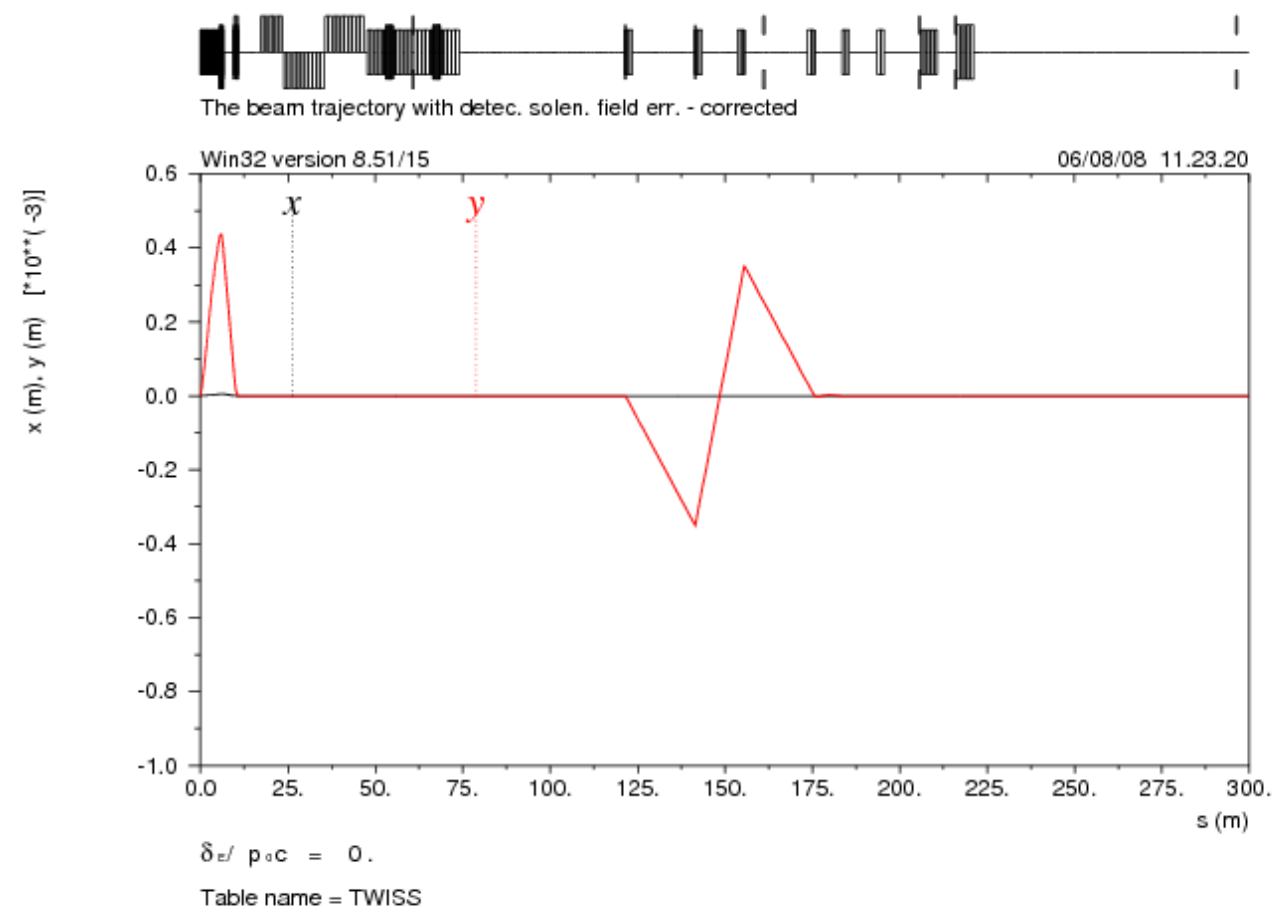

Figure 7b: Beam trajectory with detector solenoid field errors after correction. 


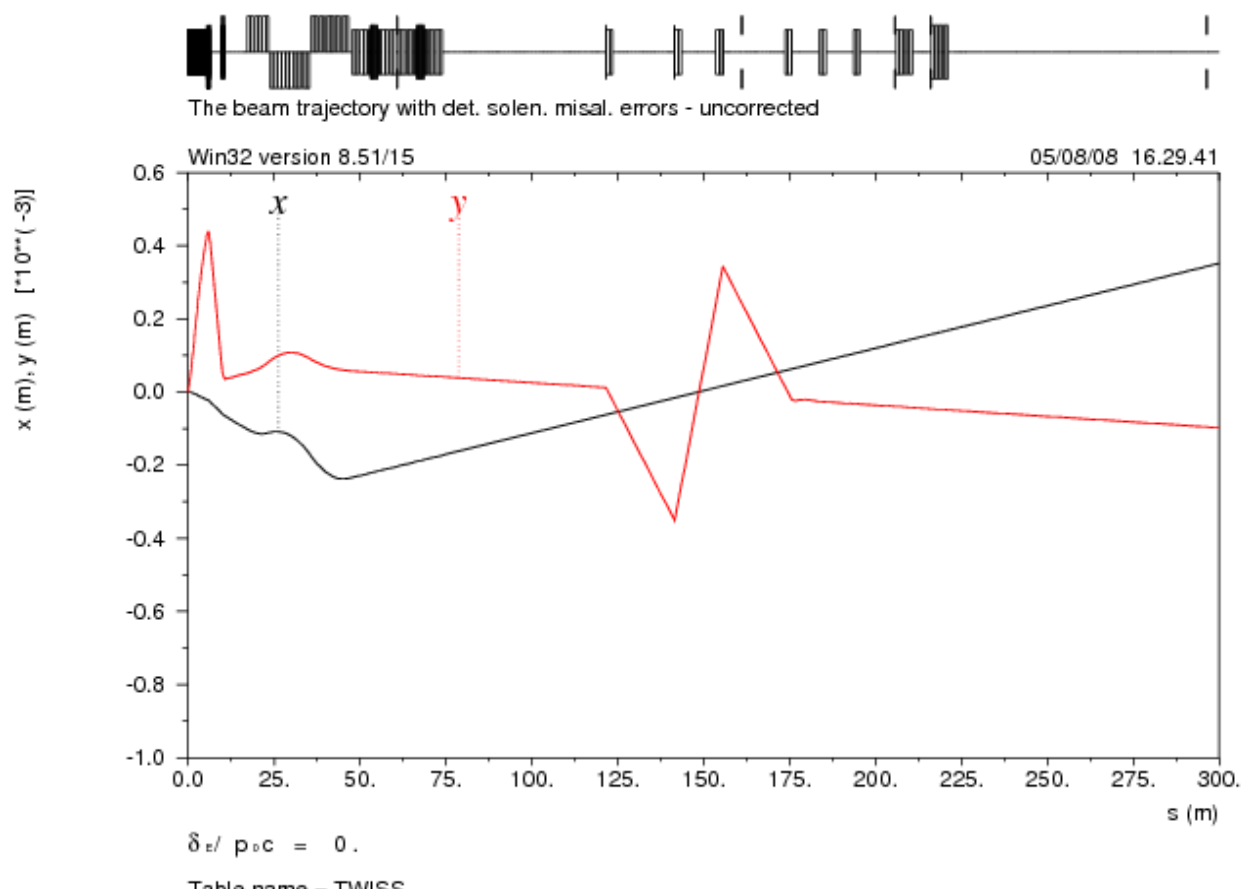

Figure 8a: Beam trajectory with detector solenoid alignment errors before correction.

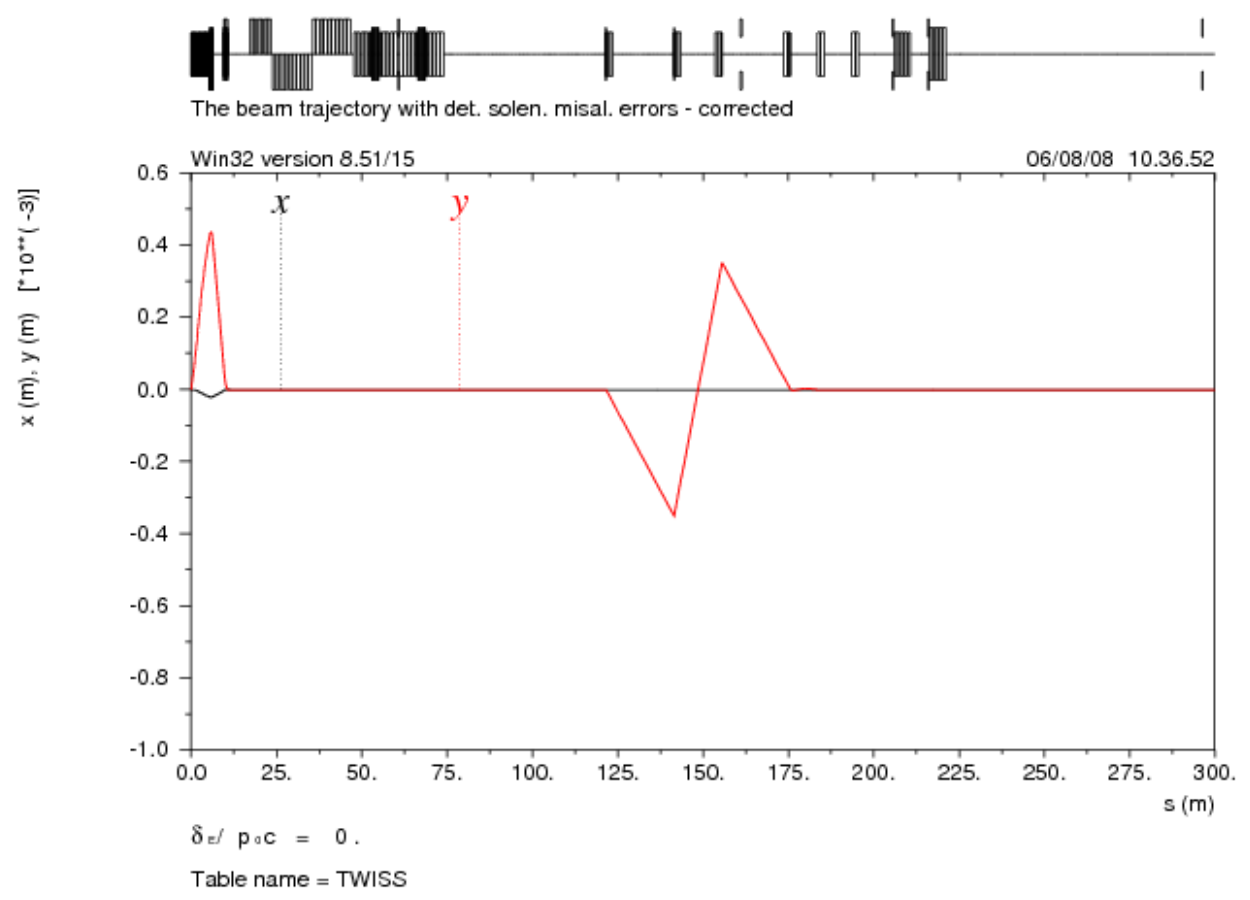

Figure 8b: Beam trajectory with detector solenoid alignment errors after correction. 
Table 5: Primary disrupted beam loss (W) in the extraction collimators for the Nominal (C11) parameter option without and with IP $y$-offset for various types of errors. Red colour - after corrections. Fast sweeping kickers are set to sweep the beam on $R=3 \mathrm{~cm}$ circle at dump.

\begin{tabular}{|l|l|l|l|l|l|}
\hline \multicolumn{7}{|c|}{$\begin{array}{c}\text { Type off } \\
\text { errors }\end{array}$} & COLE & COLCD & COLW1 & COLW2 & COLW3 \\
\hline NO Errors & 0 & 0 & 160 & 79 & 926 \\
\hline Bend Field & 0 & 0 & 160 & 79 & 1138 \\
& 1 & 0 & 160 & 79 & 1137 \\
\hline Bend & 0 & 0 & 160 & 79 & 1093 \\
Misalignment & 0 & 0 & 160 & 79 & 1019 \\
\hline Quad Field & 0 & 0 & 160 & 79 & 903 \\
& 0 & 0 & 160 & 79 & 950 \\
\hline Quad & 0 & 0 & 77 & 142 & 852 \\
Misalignment & 0 & 0 & 77 & 162 & 832 \\
\hline Solenoid & 0 & 0 & 160 & 79 & 926 \\
Field & 1 & 0 & 239 & 93 & 1323 \\
\hline Solenoid & 0 & 0 & 160 & 79 & 977 \\
Misalignment & 0 & 0 & 239 & 93 & 1286 \\
\hline
\end{tabular}

\begin{tabular}{|l|l|l|l|l|l|}
\hline \multicolumn{7}{|c|}{$\begin{array}{c}\text { C11 y-offset, } E=500 \mathrm{GeV} \text { center-of-mass } \\
\text { errors }\end{array}$} & COLE & COLCD & COLW1 & COLW2 & COLW3 \\
\hline NO Errors & 0 & 80 & 3854 & 3343 & 8920 \\
\hline Bend Field & 3 & 80 & 3854 & 3396 & 9401 \\
& 7 & 317 & 4881 & 3594 & 10221 \\
\hline Bend & 3 & 80 & 3763 & 3412 & 9039 \\
Misalignment & 3 & 80 & 3763 & 3411 & 8920 \\
\hline Quad Field & 3 & 80 & 3765 & 3344 & 8840 \\
& 3 & 80 & 3854 & 3343 & 8920 \\
\hline Quad & 3 & 80 & 3626 & 2805 & 7907 \\
Misalignment & 3 & 80 & 3626 & 3170 & 8247 \\
\hline Solenoid & 3 & 80 & 3854 & 3344 & 8920 \\
Field & 4 & 234 & 4284 & 3409 & 9323 \\
\hline Solenoid & 3 & 80 & 3660 & 3352 & 8871 \\
Misalignment & 4 & 154 & 3874 & 3410 & 9144 \\
\hline
\end{tabular}


Table 6: Primary disrupted beam loss (W) in the extraction collimators for the Low Y-emittance (C13) parameter option without and with IP $y$-offset for various types of errors. Red colour - after corrections. Fast sweeping kickers are set to sweep the beam on $R=3 \mathrm{~cm}$ circle at dump.

\begin{tabular}{|l|l|l|l|l|l|}
\hline \multicolumn{7}{|c|}{ C13, $E=500 \mathrm{GeV}$ center-of-mass } \\
\hline $\begin{array}{c}\text { Type off } \\
\text { errors }\end{array}$ & COLE & COLCD & COLW1 & COLW2 & COLW3 \\
\hline NO Errors & 0 & 66 & 1421 & 1141 & 5716 \\
\hline Bend Field & 4 & 66 & 1422 & 1142 & 5643 \\
& 7 & 66 & 1422 & 1216 & 5801 \\
\hline Bend & 4 & 66 & 1422 & 1065 & 5977 \\
Misalignment & 4 & 66 & 1422 & 1142 & 5664 \\
\hline Quad Field & 4 & 66 & 1422 & 1143 & 5692 \\
\hline Quad & 4 & 66 & 1421 & 1167 & 5615 \\
Misalignment & 4 & 66 & 1339 & 1147 & 5613 \\
\hline Solenoid & 4 & 66 & 1339 & 1147 & 5504 \\
Field & 6 & 66 & 1422 & 1142 & 5719 \\
\hline Solenoid & 4 & 66 & 1421 & 1167 & 5697 \\
Misalignment & 6 & 66 & 1339 & 1127 & 5811 \\
\hline
\end{tabular}

\begin{tabular}{|l|l|l|l|l|l|}
\hline \multicolumn{6}{|c|}{ C13 y-offset, $E=500 \mathrm{GeV}$ center-of-mass } \\
\hline $\begin{array}{c}\text { Type off } \\
\text { errors }\end{array}$ & COLE & COLCD & COLW1 & COLW2 & COLW3 \\
\hline NO Errors & 0 & 0 & 2322 & 1710 & 8494 \\
\hline Bend Field & 0 & 0 & 2323 & 1765 & 8787 \\
& 0 & 0 & 2827 & 1851 & 9066 \\
\hline Bend & 0 & 0 & 2323 & 1792 & 8779 \\
Misalignment & 0 & 0 & 2322 & 1684 & 8440 \\
\hline Quad Field & 0 & 0 & 2322 & 1684 & 8490 \\
& 0 & 0 & 2323 & 1711 & 8475 \\
\hline Quad & 0 & 0 & 2221 & 1486 & 8365 \\
Misalignment & 0 & 0 & 2221 & 1599 & 8652 \\
\hline Solenoid & 0 & 0 & 2322 & 1684 & 8548 \\
Field & 0 & 0 & 2425 & 1826 & 8621 \\
\hline Solenoid & 0 & 0 & 2323 & 1549 & 8932 \\
Misalignment & 0 & 0 & 2323 & 1711 & 8514 \\
\hline
\end{tabular}


Table 7: Primary disrupted beam loss (W) in the extraction collimators for the Low Power (C14) parameter option without and with IP $y$-offset for various types of errors. Red colour - after corrections. Fast sweeping kickers are set to sweep the beam on $R=3 \mathrm{~cm}$ circle at dump.

\begin{tabular}{|l|l|l|l|l|l|}
\hline \multicolumn{7}{|c|}{ C14, $E=500 \mathrm{GeV}$ center-of-mass } \\
\hline $\begin{array}{c}\text { Type off } \\
\text { errors }\end{array}$ & COLE & COLCD & COLW1 & COLW2 & COLW3 \\
\hline NO Errors & 104 & 966 & 10272 & 8150 & 21461 \\
\hline Bend Field & 190 & 990 & 10580 & 8363 & 22535 \\
& 249 & 1377 & 11909 & 9535 & 23301 \\
\hline Bend & 190 & 990 & 10498 & 8362 & 22825 \\
Misalignment & 190 & 990 & 10455 & 8446 & 21901 \\
\hline Quad Field & 185 & 966 & 10144 & 8148 & 21475 \\
\hline Quad & 184 & 966 & 10594 & 8186 & 21616 \\
Misalignment & 170 & 789 & 8881 & 8398 & 20986 \\
\hline Solenoid & 190 & 859 & 9309 & 8385 & 21311 \\
Field & 179 & 990 & 10529 & 8316 & 21984 \\
\hline Solenoid & 189 & 1115 & 11113 & 8638 & 22801 \\
Misalignment & 177 & 930 & 9815 & 8451 & 22128 \\
\hline
\end{tabular}

\begin{tabular}{|l|l|l|l|l|l|}
\hline \multicolumn{7}{|c|}{ C14 y-offset, $E=500 \mathrm{GeV}$ center-of-mass } \\
\hline $\begin{array}{c}\text { Type off } \\
\text { errors }\end{array}$ & COLE & COLCD & COLW1 & COLW2 & COLW3 \\
\hline NO Errors & 344 & 11528 & 63655 & 38603 & 61337 \\
\hline Bend Field & 374 & 11700 & 65208 & 39599 & 63093 \\
& 528 & 16113 & 64196 & 35353 & 62744 \\
\hline Bend & 374 & 11816 & 65189 & 39830 & 63523 \\
Misalignment & 374 & 11816 & 65347 & 39257 & 62372 \\
\hline Quad Field & 364 & 11528 & 63451 & 38628 & 61064 \\
\hline Quad & 364 & 11415 & 63825 & 38834 & 61393 \\
Misalignment & 334 & 9578 & 65250 & 40531 & 60778 \\
\hline Solenoid & 374 & 10360 & 64886 & 39596 & 61448 \\
Field & 370 & 11776 & 65177 & 39676 & 62751 \\
\hline Solenoid & 374 & 13380 & 65054 & 37450 & 62802 \\
Misalignment & 370 & 11306 & 65311 & 40794 & 63569 \\
\hline
\end{tabular}


Table 8: $\langle x\rangle$ and $\langle y\rangle$ offsets of the beam core with $|\Delta \mathbf{p} / \mathbf{p}|<1 \%$, the average spin projection $P$, and the percentage of particles $\kappa$ within laser spot at the Compton IP for Nominal (C11) beam parameter set (with and without $y$ offset) when various types of magnet errors are applied. Red colour - after correction.

\begin{tabular}{|c|c|c|c|c|}
\hline \multicolumn{5}{|c|}{ C11, $E=500 \mathrm{GeV}$ center-of-mass } \\
\hline $\begin{array}{l}\text { Type off } \\
\text { errors }\end{array}$ & $\begin{array}{l}<x> \\
{[\mu m]}\end{array}$ & $\begin{array}{l}<y> \\
{[\mu m]}\end{array}$ & $P[\%]$ & $\kappa$ \\
\hline NO Errors & 2.539 & -48.226 & 99.82 & 46.3 \\
\hline Bend Field & $\begin{array}{l}2.539 \\
2.519\end{array}$ & $\begin{array}{l}-292.889 \\
-44.702\end{array}$ & $\begin{array}{l}99.82 \\
99.82\end{array}$ & $\begin{array}{l}0.0 \\
46.6\end{array}$ \\
\hline $\begin{array}{l}\text { Bend } \\
\text { Misalignment }\end{array}$ & $\begin{array}{l}354.333 \\
-3.366\end{array}$ & $\begin{array}{l}-48.262 \\
-48.244\end{array}$ & $\begin{array}{l}99.72 \\
99.82\end{array}$ & $\begin{array}{l}0.0 \\
46.2\end{array}$ \\
\hline Quad Field & $\begin{array}{l}2.445 \\
2.676\end{array}$ & $\begin{array}{l}-47.365 \\
-47.946\end{array}$ & $\begin{array}{l}99.82 \\
99.81\end{array}$ & $\begin{array}{l}44.6 \\
47.3\end{array}$ \\
\hline $\begin{array}{l}\text { Quad } \\
\text { Misalignment }\end{array}$ & $\begin{array}{l}191.327 \\
72.437\end{array}$ & $\begin{array}{l}-435.576 \\
-169.972\end{array}$ & $\begin{array}{l}0.00 \\
99.77\end{array}$ & $\begin{array}{l}0.0 \\
10.5\end{array}$ \\
\hline $\begin{array}{l}\text { Solenoid } \\
\text { Field }\end{array}$ & $\begin{array}{l}2.515 \\
3.153\end{array}$ & $\begin{array}{l}-48.314 \\
-46.840\end{array}$ & $\begin{array}{l}99.82 \\
99.82\end{array}$ & $\begin{array}{l}46.3 \\
46.4\end{array}$ \\
\hline $\begin{array}{l}\text { Solenoid } \\
\text { Misalignment }\end{array}$ & $\begin{array}{l}5.100 \\
2.744\end{array}$ & $\begin{array}{l}12.242 \\
17.821\end{array}$ & $\begin{array}{l}99.82 \\
99.82\end{array}$ & $\begin{array}{l}46.6 \\
46.5\end{array}$ \\
\hline
\end{tabular}

\begin{tabular}{|l|l|l|l|l|}
\hline \multicolumn{5}{|c|}{ C11 y-offset, $E=500 \mathrm{GeV}$ center-of-mass } \\
\hline $\begin{array}{c}\text { Type off } \\
\text { errors }\end{array}$ & $\begin{array}{l}<x> \\
{[\mu \mathrm{m}]}\end{array}$ & $\begin{array}{l}<y> \\
{[\mu \mathrm{m}]}\end{array}$ & $P[\%]$ & $\kappa$ \\
\hline NO Errors & 2.984 & -51.474 & 99.82 & 33.1 \\
\hline Bend Field & 2.984 & -296.169 & 99.82 & 1.1 \\
& 3.318 & -47.436 & 99.82 & 33.1 \\
\hline Bend & 354.867 & -51.480 & 0.00 & 0.0 \\
Misalignment & -3.147 & -52.076 & 99.82 & 33.1 \\
\hline Quad Field & 2.834 & -50.146 & 99.82 & 32.9 \\
& 3.065 & -51.311 & 99.82 & 30.6 \\
\hline Quad & 191.810 & -440.036 & 99.79 & 0.5 \\
Misalignment & 72.735 & -173.931 & 99.79 & 9.4 \\
\hline Solenoid & 3.001 & -51.525 & 99.82 & 33.1 \\
Field & 3.549 & -49.311 & 99.82 & 33.1 \\
\hline Solenoid & 5.793 & 8.540 & 99.82 & 32.5 \\
Misalignment & 3.245 & 14.390 & 99.82 & 32.1 \\
\hline
\end{tabular}


Table 9: $\langle x\rangle$ and $\langle y\rangle$ offsets of the beam core with $|\Delta \mathrm{p} / \mathrm{p}|<1 \%$, the average spin projection $P$, and the percentage of particles $\kappa$ within laser spot at the Compton IP for Low Y-emittance (C13) beam parameter set (with and without $y$ offset) when various types of magnet errors are applied. Red colour - after correction.

\begin{tabular}{|c|c|c|c|c|}
\hline \multicolumn{5}{|c|}{ C13, $E=500 \mathrm{GeV}$ center-of-mass } \\
\hline $\begin{array}{c}\text { Type off } \\
\text { errors }\end{array}$ & $\begin{array}{l}<x> \\
{[\mu m]}\end{array}$ & $\begin{array}{l}\langle y> \\
{[\mu m]}\end{array}$ & $P[\%]$ & $\kappa$ \\
\hline NO Errors & 2.989 & -58.097 & 99.69 & 32.9 \\
\hline Bend Field & $\begin{array}{l}2.989 \\
3.363\end{array}$ & $\begin{array}{l}-302.843 \\
-53.314\end{array}$ & $\begin{array}{l}0.00 \\
99.69\end{array}$ & $\begin{array}{l}0.0 \\
33.1\end{array}$ \\
\hline $\begin{array}{l}\text { Bend } \\
\text { Misalignment }\end{array}$ & $\begin{array}{l}354.946 \\
-3.666\end{array}$ & $\begin{array}{l}-302.900 \\
-57.890\end{array}$ & $\begin{array}{l}0.00 \\
99.69\end{array}$ & $\begin{array}{l}0.0 \\
32.9\end{array}$ \\
\hline Quad Field & $\begin{array}{l}3.521 \\
2.833\end{array}$ & $\begin{array}{l}-57.351 \\
-57.602\end{array}$ & $\begin{array}{l}99.71 \\
99.68\end{array}$ & $\begin{array}{l}30.1 \\
34.4\end{array}$ \\
\hline $\begin{array}{l}\text { Quad } \\
\text { Misalignment }\end{array}$ & $\begin{array}{l}191.761 \\
72.477\end{array}$ & $\begin{array}{l}-447.284 \\
-181.024\end{array}$ & $\begin{array}{l}0.00 \\
99.58\end{array}$ & $\begin{array}{l}0.0 \\
9.6\end{array}$ \\
\hline $\begin{array}{l}\text { Solenoid } \\
\text { Field }\end{array}$ & $\begin{array}{l}3.038 \\
3.704\end{array}$ & $\begin{array}{l}-58.190 \\
-56.221\end{array}$ & $\begin{array}{l}99.69 \\
99.69\end{array}$ & $\begin{array}{l}32.9 \\
32.9\end{array}$ \\
\hline $\begin{array}{l}\text { Solenoid } \\
\text { Misalignment }\end{array}$ & $\begin{array}{l}6.073 \\
3.112\end{array}$ & $\begin{array}{l}1.717 \\
7.763\end{array}$ & $\begin{array}{l}99.70 \\
99.70\end{array}$ & $\begin{array}{l}33.2 \\
33.1\end{array}$ \\
\hline
\end{tabular}

\begin{tabular}{|l|l|l|l|l|}
\hline \multicolumn{5}{|c|}{ C13 y-offset, $E=500 \mathrm{GeV}$ center-of-mass } \\
\hline $\begin{array}{c}\text { Type off } \\
\text { errors }\end{array}$ & $\begin{array}{l}<x> \\
{[\mu \mathrm{m}]}\end{array}$ & $\begin{array}{l}<y> \\
{[\mu \mathrm{m}]}\end{array}$ & $P[\%]$ & $\kappa$ \\
\hline NO Errors & 1.276 & -59.175 & 99.76 & 27.3 \\
\hline Bend Field & 1.276 & -303.946 & 99.80 & 1.2 \\
& 0.910 & -53.278 & 99.76 & 27.2 \\
\hline Bend & 353.267 & -304.002 & 99.62 & 0.3 \\
Misalignment & -5.371 & -59.592 & 99.76 & 27.2 \\
\hline Quad Field & 0.782 & -59.358 & 99.77 & 26.7 \\
\hline Quad & 1.059 & -58.268 & 99.75 & 24.2 \\
Misalignment & 189.565 & -447.024 & 99.74 & 0.6 \\
\hline Solenoid & 70.819 & -181.815 & 99.73 & 9.0 \\
Field & 0.998 & -59.311 & 99.76 & 27.3 \\
\hline Solenoid & 1.839 & -57.252 & 99.76 & 27.3 \\
Misalignment & 4.231 & 0.639 & 99.77 & 26.7 \\
\hline
\end{tabular}


Table 10: $\langle x\rangle$ and $\langle y\rangle$ offsets of the beam core with $|\Delta \mathrm{p} / \mathbf{p}|<1 \%$, the average spin projection $P$, and the percentage of particles $\kappa$ within laser spot at the Compton IP for Low Power (C14) beam parameter set (with and without $y$ offset) when various types of magnet errors are applied. Red colour - after correction.

\begin{tabular}{|c|c|c|c|c|}
\hline \multicolumn{5}{|c|}{ C14, $E=500 \mathrm{GeV}$ center-of-mass } \\
\hline $\begin{array}{l}\text { Type off } \\
\text { errors }\end{array}$ & $\begin{array}{l}<x> \\
{[\mu m]}\end{array}$ & $\begin{array}{l}\langle y> \\
{[\mu m]}\end{array}$ & $P[\%]$ & $\kappa$ \\
\hline NO Errors & 2.372 & -54.113 & 99.17 & 72.7 \\
\hline Bend Field & $\begin{array}{l}2.372 \\
2.248\end{array}$ & $\begin{array}{l}-298.810 \\
-50.035\end{array}$ & $\begin{array}{l}99.13 \\
99.15\end{array}$ & 72.6 \\
\hline $\begin{array}{l}\text { Bend } \\
\text { Misalignment }\end{array}$ & $\begin{array}{l}354.257 \\
-4.049\end{array}$ & $\begin{array}{l}-54.118 \\
-54.029\end{array}$ & $\begin{array}{l}99.13 \\
99.17\end{array}$ & 72.8 \\
\hline Quad Field & $\begin{array}{l}2.866 \\
2.418\end{array}$ & $\begin{array}{l}-52.947 \\
-54.027\end{array}$ & $\begin{array}{l}99.17 \\
99.17\end{array}$ & $\begin{array}{l}74.6 \\
71.7\end{array}$ \\
\hline $\begin{array}{l}\text { Quad } \\
\text { Misalignment }\end{array}$ & $\begin{array}{l}191.061 \\
72.994\end{array}$ & $\begin{array}{l}-442.516 \\
-176.537\end{array}$ & $\begin{array}{l}99.15 \\
99.17\end{array}$ & 91.2 \\
\hline $\begin{array}{l}\text { Solenoid } \\
\text { Field }\end{array}$ & $\begin{array}{l}2.379 \\
3.177\end{array}$ & $\begin{array}{l}-54.187 \\
-52.093\end{array}$ & $\begin{array}{l}99.17 \\
99.16\end{array}$ & $\begin{array}{l}72.7 \\
72.7\end{array}$ \\
\hline $\begin{array}{l}\text { Solenoid } \\
\text { Misalignment }\end{array}$ & $\begin{array}{l}4.717 \\
2.815\end{array}$ & $\begin{array}{l}5.979 \\
12.108\end{array}$ & $\begin{array}{l}99.17 \\
99.17\end{array}$ & $\begin{array}{l}72.6 \\
72.8\end{array}$ \\
\hline
\end{tabular}

\begin{tabular}{|l|l|l|l|l|}
\hline \multicolumn{5}{|c|}{ C14 y-offset, $E=500 \mathrm{GeV}$ center-of-mass } \\
\hline $\begin{array}{c}\text { Type off } \\
\text { errors }\end{array}$ & $\begin{array}{l}<x> \\
{[\mu \mathrm{m}]}\end{array}$ & $\begin{array}{l}<y> \\
{[\mu \mathrm{m}]}\end{array}$ & $P[\%]$ & $\kappa$ \\
\hline NO Errors & -1.216 & -59.196 & 98.34 & 83.0 \\
\hline Bend Field & -0.570 & -304.386 & 98.44 & 80.5 \\
& -0.842 & -55.118 & 98.28 & 83.0 \\
\hline Bend & 350.767 & -59.201 & 98.44 & 80.0 \\
Misalignment & -7.578 & -58.938 & 98.35 & 83.0 \\
\hline Quad Field & -1.311 & -58.047 & 98.36 & 84.2 \\
\hline Quad & -0.341 & -59.236 & 98.36 & 85.4 \\
Misalignment & 187.896 & -447.487 & 98.48 & 87.2 \\
\hline Solenoid & 68.832 & -181.067 & 98.44 & 93.7 \\
Field & -1.044 & -59.499 & 98.35 & 83.0 \\
\hline Solenoid & -0.234 & -57.188 & 98.32 & 83.0 \\
Misalignment & 2.467 & 0.186 & 98.36 & 83.3 \\
\hline
\end{tabular}




\section{ACKNOWLEDGEMENT}

We acknowledge that this study was originally started and funded at the Cockcroft Institute, University of Manchester, UK, under the leadership of Dr. Deepa Angal-Kalinin and Dr. Robert Appleby. We thank them for the initial guidance and helpful discussions. This work is supported by the U.S. Department of Energy contract DE-AC02$76 S F 00515$.

\section{References}

[1] J. Brau (Ed.) et al., "ILC Reference Design Report: ILC Global Design Effort and World Wide Study”, FERMILAB-APC (2007).

[2] MAD-8 program, http://mad.web.cern.ch/mad/mad8web/mad8.html .

[3] DIMAD program, http://www-project.slac.stanford.edu/lc/local/AccelPhysics/dimad.pdf .

[4] Y. Nosochkov et al., "14 mrad Extraction Line Optics for Push-Pull”, SLAC-PUB-12856, LCWS-2007-MDI13 (2007).

[5] B. Parker et al., "The Superconducting Magnets of the ILC Beam Delivery System”, PAC07-THPMS091, SLAC-PUB-12832 (2007).

[6] SiD Design Study, http://silicondetector.org/display/SiD/home .

[7] T. Behnke (Ed.) et al., "ILC Reference Design Report Volume 4 - Detectors”, FERMILAB-APC (2007).

[8] D. Toprek, Y. Nosochkov, "Evaluation and Compensation of Detector Solenoid Effects on Disrupted Beam in the ILC 14 mrad Extraction Line”, SLAC-PUB-13492 (2008).

[9] K. Moffeit et al., "Proposal to Modify the Polarimeter Chicane in the ILC 14 mrad Extraction Line", SLACPUB-12425, IPBI-TN-2007-1 (2007).

[10] D. Schulte, "Study of Electromagnetic and Hadronic Background in the Interaction Region of the TESLA Collider”, PhD. Thesis, TESLA-97-08 (1996).

[11] T. Raubenheimer, “Suggested ILC Beam Parameter Range, Rev. 2/28/05”, http://wwwproject.slac.stanford.edu/ilc/acceldev/beampar/Suggested\%20ILC\%20Beam\%20Parameter\%20Space.pdf (2005).

[12] A. Seryi, http://www.slac.stanford.edu/ seryi/ILC_new_gp_files/ (2005).

[13] Y. Nosochkov, T.O. Raubenheimer, K.A. Thompson, "NLC beam properties and extraction line performance with beam offset at IP”, SLAC-PUB-8872, PAC-2001-FPAH065 (2001).

[14] M. Woods et al., "Luminosity, energy and polarization studies for the linear collider: Comparing e+ e- and e-efor NLC and TESLA", SLAC-PUB-10353, IPBI-TN-2004-1 (2004).

[15] P.C. Rowson, M. Woods, "Experimental issues for precision electroweak physics at a high luminosity Z factory”, SLAC-PUB-8745 (2000). 